Verlässliche Beratung zur Existenzgründung

Was manchen Arzt vor einer Niederlassung zurückschrecken lässt, ist die Frage, ob sich die Investition in die Praxis am Ende auch rechnet, und ob der Kaufpreis durch die Einnahmen aufgebracht werden kann.

Für diese Fälle hat die Deutsche Apotheker- und Ärztebank zusammen mit dem Zentralinstitut für die kassenärztliche Versorgung das Beratungstool INKO entwickelt. Mit Hilfe dieses Programms lässt sich über Modellrechnungen überprüfen, ob sich die Vorstellungen des Existenzgründers im Praxis- und Privatbereich realisieren lassen und ob die erforderlichen Mindesteinnahmen in der übernommenen Praxis realisierbar sind. Sowohl die Investitionen, die Finanzierung als auch die voraussichtlichen Kosten im Praxis- und Privatbereich fließen in die Rechnung mit ein. So lässt sich feststellen, ob das Vorhaben wirtschaftlich tragfähig ist beziehungsweise an welchen Stellschrauben noch gedreht werden muss.

Weitere ausführliche Informationen zum Beratungstool INKO finden Sie unter: www.apobank.de

lich müsse es zwischen den Partnern natürlich stimmen.

In einer fachgleichen BAG könnte durch die Wahl eines Partners mit anderen Tätigkeitsschwerpunkten das Praxisspektrum erweitert werden, etwa für das Angebot von IGeL. Das gelte für Schwerpunkte wie Akupunktur, Chirotherapie oder Psychosomatik.

Ein häufig gewählter Ansatz in einer fachübergreifenden BAG sei es, die Versorgung aus einer Hand anzubieten, etwa Hausarzt und Diabetologe zur ganzheitlichen Betreuung von Diabetikern. Für Fachrichtungen, bei denen es geringere fachliche Überschneidungen gibt, seien eher eine Teil-Berufsausübungsgemeinschaft oder eine überörtliche BAG geeignet. In einer Teil-BAG könne zum Beispiel ein Orthopäde ein- oder zweimal im Monat eine Sprechstunde in der Kinderarztpraxis für die Vorsorgeuntersuchung zur Hüftfehlstellung anbieten. Für Eltern bringe das erhebliche Erleichterungen bei der Planung dieses zusätzlichen Arztbesuchs. Leutloff: „Die höhere Versorgungsqualität bringt gleichzeitig einen Marketingeffekt für die Praxis mit sich." Hauke Gerlof

\title{
Partnerverträge: Drum prüfe, wer sich ewig bindet ...
}

\begin{abstract}
Probezeit für Kooperationen, Haftung für Altverbindlichkeiten - die Gründung einer Kooperation oder der Eintritt in eine bestehende Gemeinschaft wirft viele Rechtsfragen auf. Entscheidend ist ein gut formulierter Vertrag.
\end{abstract}

$F_{\text {is }}^{\mathrm{u}}$ detse ur den gestandenen Medizinrechtler ist das ein Graus: „Ein paar Musterverträge nehmen, die wichtigsten Passagen zusammenschreiben, dann ist das Ding erledigt? Das wäre ja fast so, als würde ich mir als Jurist den Pschyrembel durchlesen, dann das OP-Besteck zur Hand nehmen und losoperieren!" Rechtsanwalt Dr. Bernd Halbe aus Köln, Netzwerkpartner der apoBank, weiß, wie wichtig kleine Details in der Ausgestaltung von Kooperationen sein können. Um so mehr Augenmerk sollten die zukünftigen Partner einer Gesellschaft auf eine präzise Formulierung des Vertrags legen - und dafür auch die Expertise von Steuerberater, Rechtsanwalt und eventuell auch von Sachverständigen zur Bewertung von Arztpraxen hinzuziehen. „Der Einstieg in eine Kooperation ist ein bisschen so wie der Beginn einer Ehe“, sagt auch Gesundheitsökonom Jens Leutloff von der apoBank. Da sei es gut, von Anfang an zu wissen, woran man ist - von der Bewertung der Praxis bis hin zu klaren Formulierungen, welche Regelungen im Fall einer Auflösung der Gesellschaft oder bei der Kündigung durch einen Partner gelten soll.

Dabei gibt es große Unterschiede, je nachdem, ob sich mehrere Partner zu einer neuen Gemeinschaft zusammenschließen, oder ob ein Arzt in eine bestehende Kooperation eintritt, so Halbe: „Bei einer bestehenden Gemeinschaft ist meist eine Probezeit im Rahmen eines Anstellungsverhältnisses sinnvoll." Erst im zweiten Schritt erfolgt dann der Einstieg als Gesellschafter mit Kapitalbeteiligung. Das GKV-VStG habe genau das durch die Möglichkeit der Rückumwandlung einer Arztstelle in eine Zulassung erleichtert. Entsteht dagegen eine neue Kooperation, dann gehe es für die beteiligten Ärzte zunächst vor allem um die Sicherung der Investition. „Das heißt, für die Dauer von drei, vier oder fünf Jahren sollte eine Kündigung vertraglich ausgeschlossen werden."

Ganz wichtig vor Eintritt in eine bestehende Kooperation ist die genaue Klärung der Verpflichtungen der Gesellschaft aus Verträgen sowie der Kapitalverhältnisse. Dabei könnten nach aktu-

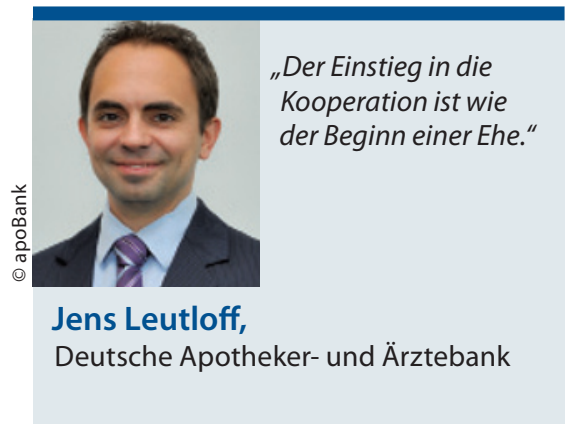

eller Rechtsprechung auch Altverbindlichkeiten für neue Gesellschafter böse Überraschungen bringen.

Schon beim Start der Gemeinschaft sollten die Modalitäten des Ausstiegs genau festgelegt sein, betont Halbe. Gerade bei einer Trennung könnten leicht fünf- bis sechsstellige Summen verschenkt werden, wenn ein Partner nicht aufmerksam ist.

Nicht zuletzt müssten auch Fragen der Vertretung bei Krankheit geregelt werden: Wer trägt die Kosten, die Gesellschaft oder der kranke Partner? Und bei Ausscheiden wird bei drohendem Ärztemangel immer wichtiger: Wer trägt das Risiko, falls kein neuer Partner gefunden wird? Eine Kooperation, resümiert Halbe, sei immer ein lebendes Gebilde. Verträge müssten daher „Raum für Veränderungen lassen“, etwa bei der Gewinn- oder der Arbeitsverteilung.

Hauke Gerlof 